\title{
Propagation characteristics of wideband high-speed railway channel in viaduct scenario at $2.35 \mathrm{GHz}$
}

\author{
Yunling GUO ${ }^{1 *}$, Jianhua ZHANG ${ }^{1}$, Cheng TAO ${ }^{2}$, Liu LIU², Lei TIAN ${ }^{1}$ \\ 1. Key Laboratory of Universal Wireless Communications (Ministry of Education), Wireless Technology Innovation Institute, Bei- \\ jing University of Posts and Telecommunications, Beijing 100876, China \\ 2. Institute of Broadband Wireless Mobile Communications, Beijing Jiaotong University, Beijing 100044, China
}

\begin{abstract}
In order to obtain accurate characteristics of wireless channels in the viaduct area of China, a channel measurement was taken in a railway viaduct scenario of the Zhengzhou-Xi' an passenger dedicated line with a bandwidth of $50 \mathrm{MHz}$ at $2.35 \mathrm{GHz}$. The single-slope log-distance model is used to analyze the path-loss (PL), and the distribution of shadow fading (SF) is obtained by statistical methods, which shows that the normal distribution fits the samples well. Ricean $K$-factor is analyzed by the method of moments, and the variation of $K$-factor is given along the measured route. Small scale such as delay spread and Doppler behavior are parameterized. Based on empirical channel measurement, this paper provides parameters for the evaluation and simulation work on viaduct scenarios of high-speed railway.
\end{abstract}

Key words: high-speed railway; channel measurement; viaduct; path-loss; delay spread; Ricean $K$-factor; Doppler

(C) 2012 JMT. All rights reserved.

\section{Introduction}

$\mathrm{T}$ o supply a high quality of service (QoS) on speech communication and data transmission for people taking high-speed trains, attention has been paid to the characteristics of the wireless channel in many railway scenarios because the characteristics of radio wave propagation have a significant impact on the QoS of the communication system. Among these scenarios, the study of radio propagation in viaduct areas becomes a central issue. A viaduct area that is built high above the ground in a railway line can not only save land resource and reduce the effect of the ground environment on railway route selection, but also ensure the safe running of high-speed trains.

In recent years, a lot of research has focused on the measurement of high-speed railway channel. In WINNER II channel models [1], measurements were conducted with a bandwidth of $120 \mathrm{MHz}$ at the center frequency of $5.2 \mathrm{GHz}$ using the RUSK sounder in a bridge-to-car scenario and in a fast train scenario on the railway line between Siegburg and Frankfurt in Germany. One transmitting antenna mounted at roof-top of the carriage and a uniform circular array (UCA) with 16

Received Oct. 21, 2012; revision accepted Nov. 9, 2012

${ }^{*}$ Corresponding author. E-mail: guoyl@bupt.edu.cn (Y.L. GUO)

(C) 2012 JMT. All rights reserved

doi: 10.3969/j.issn.2095-087X.2012.04.003 vertically polarized disc-cone elements were used in the fast train measurements. A path-loss (PL) model and the distribution of shadow fading (SF) were given in both scenarios. Delay spread characteristics were obtained in the fast train measurements and angle information as well as Ricean $K$-factor was provided in the bridge-tocar scenario. Parviainen et al. [2] carried out measurements in Taiwan with a bandwidth of $50 \mathrm{MHz}$ at $2.5 \mathrm{GHz}$, and presented high-speed train propagation characteristics, such as delay spread characteristics, angle information, and Doppler behavior. Wei et al. [3] and $\mathrm{Lu}$ et al. [4] provided a PL model in viaduct and plain scenarios and compared it with the Hata model. Wei et al. [5] obtained the distribution and autocorrelation of the SF in a viaduct scenario in the BeijingShanghai high-speed railway with a speed of $220 \mathrm{~km} / \mathrm{h}$. The result showed that the autocorrelation of SF was better modeled by the double exponential model than the exponential model. Lu et al. [6] provided the fitted PL model, SF, and dynamic range of the small scale fading of cutting scenario and compared the results to those in a viaduct scenario. The PL exponent in terrain cutting was found to be greater than that in a viaduct area. He et al. [7-9] presented an empirical PL model based on different transmit antenna height in a viaduct scenario, proposed models covering the crown width and bottom width of the cutting, and obtained the Ricean $K$-factor changing with distance. The specification on the PL model and small scale fading properties 
such as $K$-factor, Doppler frequency feature and time delay spread in a viaduct in China with a bandwidth of $10 \mathrm{MHz}$ at center frequency of $2.35 \mathrm{GHz}$ can be found in Ref. [10].

However, Refs. [3-9] employed track-side base stations of the GSM-R network at about $930 \mathrm{MHz}$ with a bandwidth of $200 \mathrm{kHz}$. These measurements cannot obtain accurate characteristics of the wideband wireless channel. WINNER II provided channel parameters in detail, but its measurements were performed in Germany at $5.2 \mathrm{GHz}$ and some channel parameters were calculated by the measured data in a bridge-to-car scenario. The results of Ref. [2] were channel parameters of small scale and focused on Doppler frequency shift. Ref. [10] showed some channel parameters in an open viaduct scenario where there were nearly no trees or other large objects around the viaduct.

Therefore, in this paper, to obtain more accurate characteristics of wireless channels in the viaduct area of China, a measurement has been carried out in a viaduct where there are many trees around in the Zhengzhou$\mathrm{Xi}$ 'an passenger dedicated high-speed railway with a bandwidth of $50 \mathrm{MHz}$ at $2.35 \mathrm{GHz}$.

A PL model with log-normal shadowing is built. PL exponent and SF standard deviation along with its distribution are discussed. Root-mean-square (RMS) delay spread with the number of the resolvable multipath components and their distribution will be analyzed at the same time. Ricean $K$-factor as function of distance and its cumulative distribution function (CDF) will be given. At last, Doppler frequency shift is counted and verified.

\section{Measurement system}

In our measurement, a setup with a transmitting antenna and a receiving antenna was used, and the Elektrobit PropSound Channel Sounder [11] was employed. The parameters of the measurement system are listed in Table 1.

Table 1 Parameters of measurement system

\begin{tabular}{cc}
\hline Items & Settings \\
\hline Center frequency & $2.35 \mathrm{GHz}$ \\
Bandwidth & $50 \mathrm{MHz}$ \\
Code length & 127 \\
Antenna type of TX & Dipole \\
The height of TX & $12.8 \mathrm{~m}$ (height from ground) \\
North latitude of TX & 34.5334 (degree) \\
East longitude of TX & 109.8313 (degree) \\
Height of RX & $9 \mathrm{~m}$ (height from ground) \\
Transmitting power & $30.8 \mathrm{~dB} \cdot \mathrm{m}$ \\
\hline
\end{tabular}

A vertical-polarized dipole excites the channel at the transmitter (TX) side, which is on the top of an 8-mhigh building. The TX antenna, whose location was recorded by a global positioning system (GPS) device, was about $12.8 \mathrm{~m}$ from the ground surface. An existing vertically-polarized wideband antenna mounted on the rooftop of the carriage was used as the receiver (RX) which was about $9 \mathrm{~m}$ high from the ground surface. A pseudo-random sequence with a length of 127 was generated at the transmitter with a chip rate of $25 \mathrm{MHz}$. Channel impulse responses (CIR) were obtained by slide-correlating the received signal with a synchronized copy of the sequence at RX. TX and RX were synchronized by a GPS clock during the measurement. Fig. 1 shows the working principle of the measurement system.

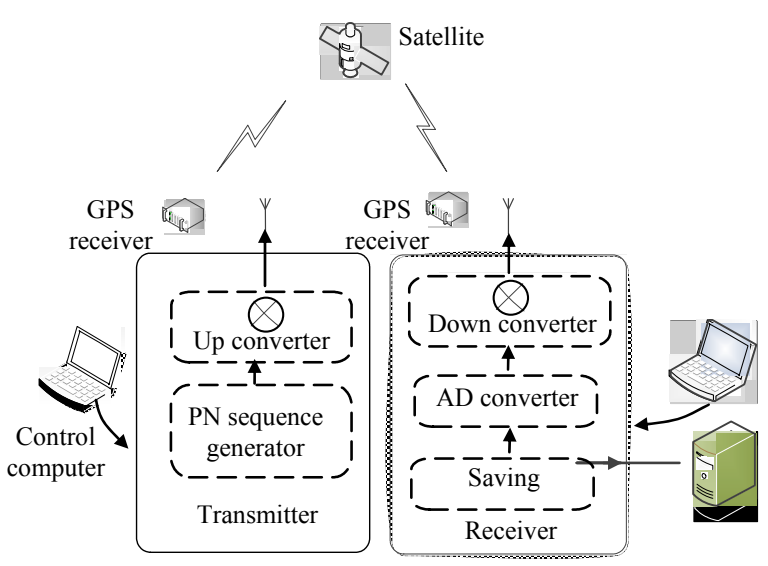

Fig. 1 Working principles of the PropSound channel sounder

\section{Measurement environment}

The channel measurement campaign is performed in the Zhengzhou-Xi'an high-speed railway with a total length of $458 \mathrm{~km}$. The highest speed of train is up to $340 \mathrm{~km} / \mathrm{h}$ in the viaduct scenario which occupies more than fifty percent of all the scenarios in the ZhengzhouXi'an railway line. Fig. 2 gives a bird-eye view of the measurement scenario, where the yellow line is the route that the train traveled.

Fig. 3 shows a typical viaduct scenario. It can be seen that there are a lot of trees between the TX and the railway. The train speed was about $190-196 \mathrm{~km} / \mathrm{h}$ when the

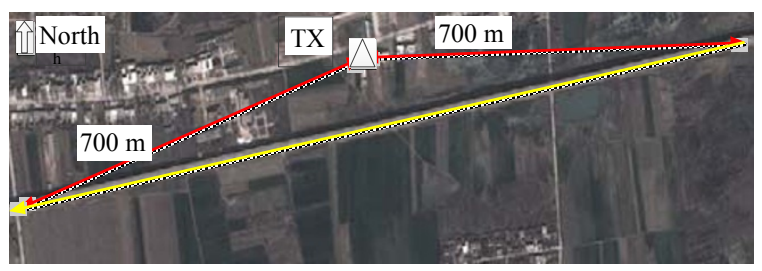

Fig. 2 Bird-eye view of the measurement environment 


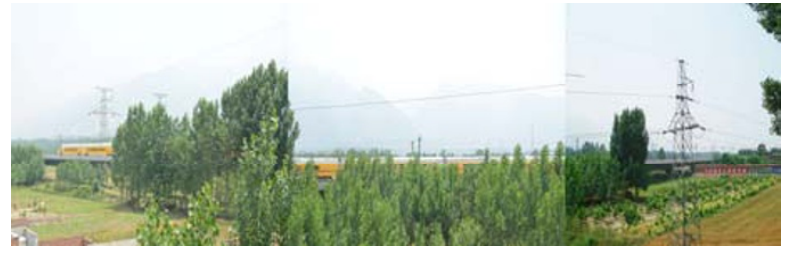

Fig. 3 Propagation environment view from TX

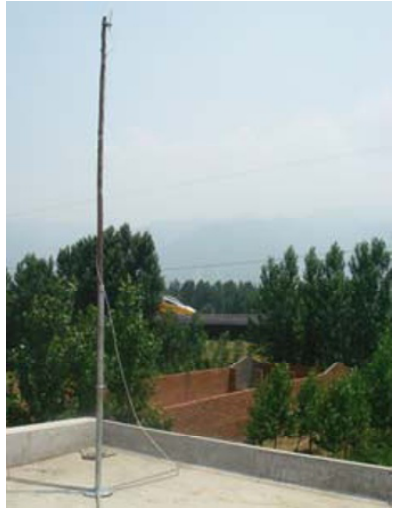

(a)

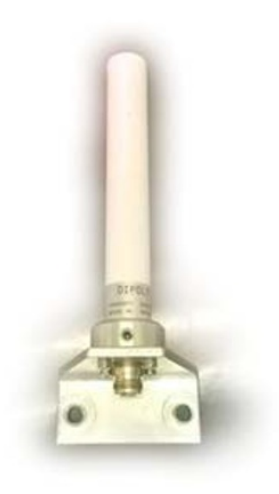

(b)
Fig. 4 Environment around TX (a) and dipole antenna used at TX (b)

measurement was carried out. The environment around the TX and the antenna used at TX are shown in Fig. 4.

\section{Results and analysis}

Large scale parameters reflect channel characteristics within a local area (LA), where only small scale fading takes place. The LA is defined as a disk with a radius of $10 \lambda$, where $\lambda$ is the wavelength, corresponding to $1.28 \mathrm{~m}$ at $2.35 \mathrm{GHz}$. As illustrated in Fig. 5, each channel sample is located in the center of a corresponding LA. Hence, a sequence of LAs can be counted from a measurement route.

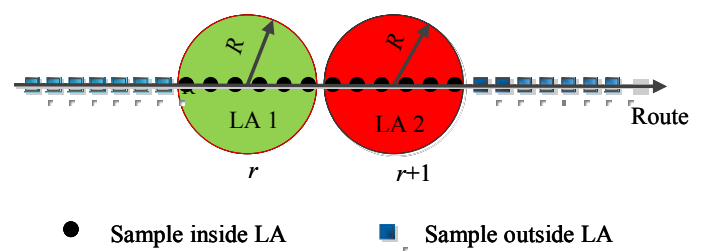

Fig. 5 Illustration of LA

\subsection{PL and SF}

A PL sample is extracted from an LA. We let $A(r, R)=\left(s_{1}, s_{2}, \cdots, s_{N}\right)$ [12] be the set of measurement position within the LA centered at $r$ with the radius $R$, where $s_{n}$ denotes the position of the $n$th sample in the LA, $n=1, \cdots, N$ and $r$ is the location of the RX. The joint effect of PL, SF and antenna gain is calculated as follows [13-14]:

$$
p(r)=\frac{1}{N} \sum_{n=1}^{N} \sum_{\tau}\left|h\left(\tau ; s_{n}\right)\right|^{2}, s_{n} \in A(r, R),
$$

where $h\left(\tau ; s_{n}\right)$ is the $n$th CIR from the raw data. The measured CIR at position $r$ can be expressed as

$$
h(\tau ; r)=\sqrt{E(r)} h_{\text {norm }}(\tau ; r),
$$

where $h_{\text {norm }}(\tau ; r)$ is the component with unitary average power and $E(r)$ is the power of the valid multipath of $h(\tau ; r)$. Let $P_{\mathrm{dB}}(r)$ be the expression of $p(r)$ in decibels. Then $P_{\mathrm{dB}}(r)$ can be written as

$$
P_{\mathrm{dB}}(r)=G_{A}-L(r)-S(r),
$$

where $G_{A}, L(r)$, and $S(r)$ are antenna gain, PL and SF, respectively, all in $\mathrm{dB}$.

The single-slope log-distance (LD) model [15] is used to simulate the PL samples. The LD model can be expressed as

$$
L(d)=A+10 n \lg d,
$$

where $d$ is the distance between the TX and RX, in $\mathrm{m}$, $n$ is the exponent of PL, and $A$ is the intercept. $P_{\mathrm{dB}}(r)$ can be calculated by the CIR using (1). Linear regression is employed to estimate the two parameters in (4) under the minimum RMS error. Hence, $S(r)$ can be obtained by Eq. (3). In the end, the distribution of SF can be obtained by statistical methods.

The measured PL samples, fitting the PL model, and the PL model of the high-speed railway in WINNEER II with frequency correction and free space model are shown in Fig. 6. The fitted PL model is

$$
L(d)=25.3+35.6 \lg d \text {, with } \sigma=4.8 \mathrm{~dB} .
$$

Ref. [10] presented a PL model with its matching parameter expressed as

$$
L(d)=12.4+30.3 \lg d \text {, with } \sigma=2.0 \mathrm{~dB} .
$$

The results of the matching parameters are different. The estimated PL exponents are 3.03 in Ref. [10] and 3.56 in this paper. The reason why the PL exponent in this paper is greater than the one of the fitted model in Ref. [10] is that the distance between the heights of transmitting and receiving antenna in the vertical direction in this paper is lower than that in Ref. [10], and the surrounding environment in this paper intensifies the effect. This phenomenon can explain bigger variance of the shadow fading in this paper. The PL exponent of the fit- 
ted curve is too high, compared to that in the free space model.

This kind of deviation from fast train measurements is also shown in WINNEER II, in which the PL exponent is 4.11. The values of PL samples are greater than the values of the free space model and similar to the values from the fast train measurements in WINNEER II. It is concluded that the extraordinary behavior of the propagation curve depends on the special circumstances in the measurement. The antenna used in the carriage roof of the train is a vertical-polarized wideband antenna. It is mounted very near the roof that acts as a ground plane. This will lead to a null in the radiation pattern in the surface containing the ground plane. Then the environment around the high-speed railway will intensify the effect. Finally, as mentioned above, the TX antenna of the measurement equipment is quite low compared to the height of receiving antenna. However, the values of the PL model in Ref. [10] are too small compared with the measured PL samples when the distance from TX to RX is $100-600 \mathrm{~m}$. Fig. 7 shows the distribution of SF. One can see that the normal distribution fits the samples well.

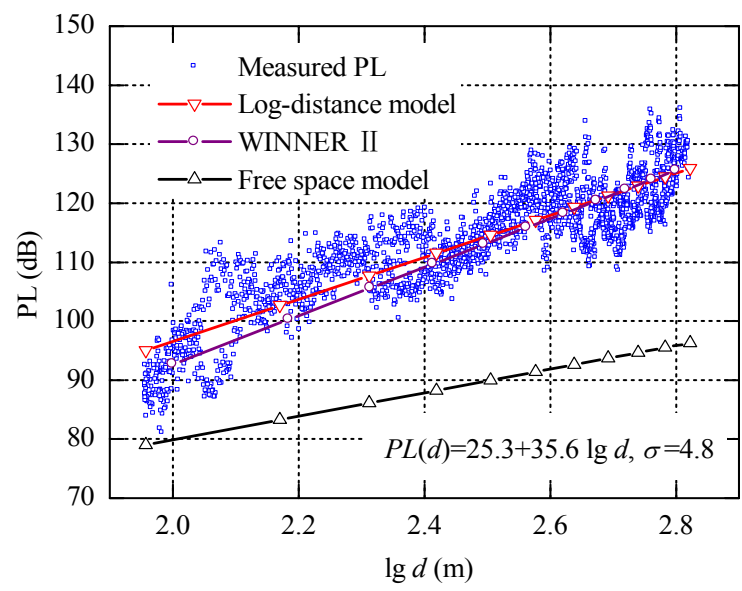

Fig. 6 PL samples and corresponding model of a viaduct

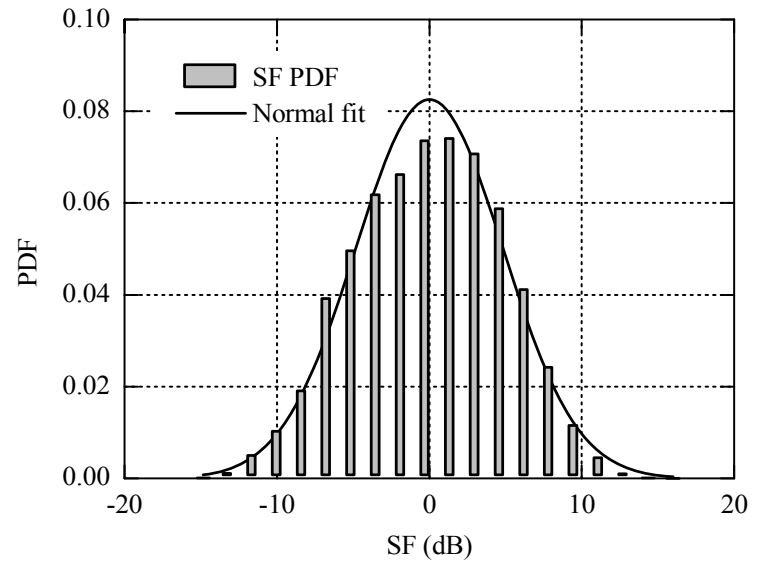

Fig. 7 Probability density function (PDF) of SF in a viaduct

\subsection{Delay spread}

A signal can travel from TX to RX via different paths. Multipath propagation leads to dispersion in the delay domain. Thus CIRs have different values at different delays. This dispersion has an impact on the design and performance of the communication system.

The delay of the measured CIRs is the absolute transmitted delay, but we can still get RMS delay $\operatorname{spread} \tau_{\text {rms }}$ from CIRs. Let $X(r)(r=1,2, \cdots, N)$ be the $r$ th sample of the power delay profile (PDP) at a certain time, where PDP can be calculated by $X(r, \tau)=\|h(r, \tau)\|^{2}$.

$\tau_{\text {rms }}$ is the second moment of the PDP and can be obtained by the following formula [16-18]:

$$
\tau_{\mathrm{rms}}=\sqrt{\frac{\sum_{r=1}^{N} \tau_{r}^{2} X(r)}{\sum_{r=1}^{N} X(r)}-\left(\frac{\sum_{r=1}^{N} \tau_{r} X(r)}{\left.\sum_{r=1}^{N} X(r)\right)^{2}},\right.}
$$

where $\tau_{r}$ denotes the excess delay of the $r$ th sample of PDP.

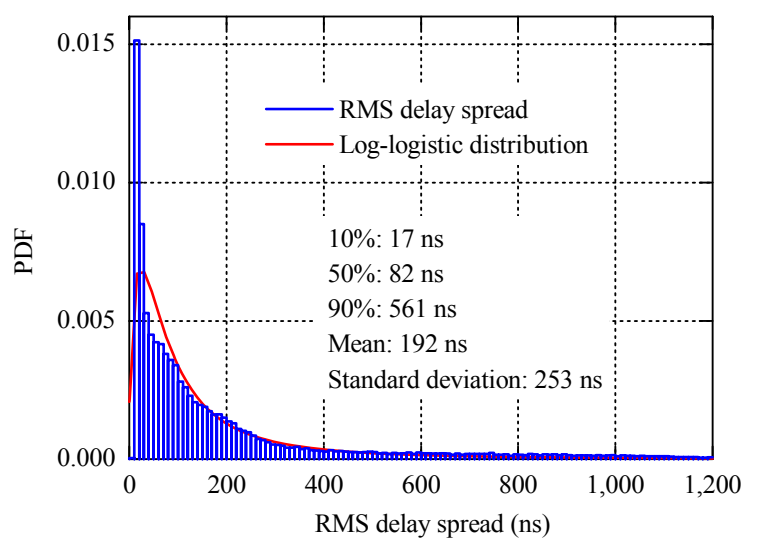

Fig. 8 PDF of RMS delay spread in a viaduct

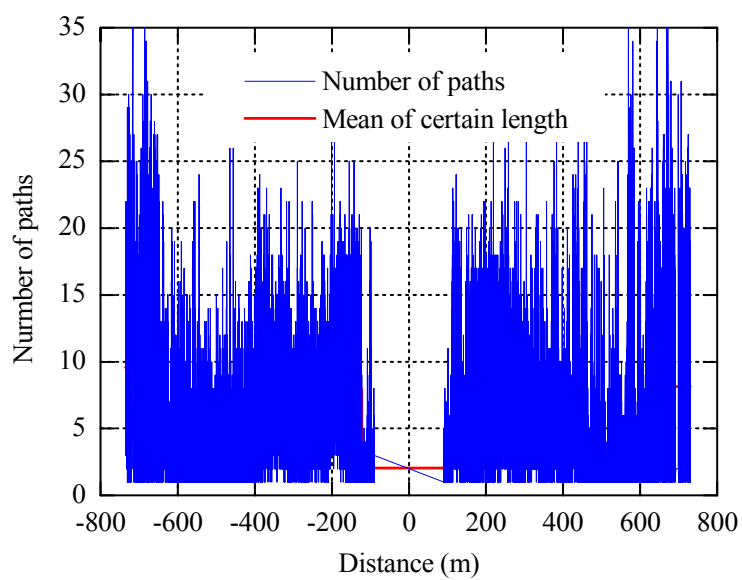

Fig. 9 The number of the resolvable multipath components changes with the distance between TX and RX 


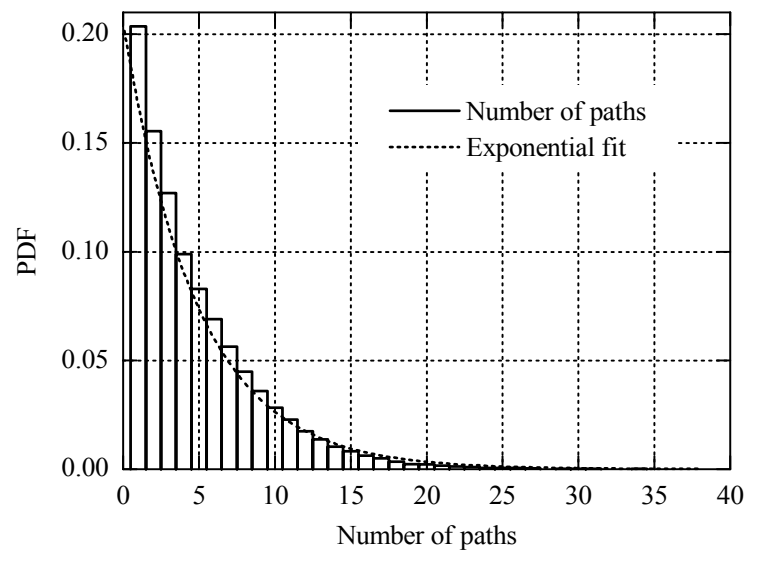

Fig. 10 PDF of the number of the resolvable multipath components in a viaduct scenario

Fig. 8 shows the probability density function (PDF) of RMS delay spread in a viaduct scenario. The mean of the calculated RMS delay spread is greater than that in WINNER II, where the value is $40 \mathrm{~ns}$ for line-of-sight (LOS) case and $55 \mathrm{~ns}$ for non-line-of-sight (NLOS) case which are calculated by the measurement in rural macrocell scenario at $5.25 \mathrm{GHz}$. Long measurement distance and the surrounding environment of the measured viaduct can lead to a bigger RMS delay spread. Fig. 9 shows the number of resolvable multipath components along the measured route, while Fig. 10 shows the PDF of the number of resolvable multipath components.

\subsection{Ricean K-factor}

The Ricean $K$-factor is defined as the average power ratio of the fixed and multipath components. It can be estimated by the method of Ref. [19]. Before getting the $K$ factor, the measured CIRs should be transformed into the narrow band form [12]. The narrow band form $g(r)$ can be written as

$$
g(r)=\sum_{\tau} h_{\text {norm }}(\tau ; r) .
$$

The Ricean $K$-factor is then given by

$$
K(r)=\frac{\sqrt{G_{a}^{2}(r)-G_{v}^{2}(r)}}{G_{a}(r)-\sqrt{G_{a}^{2}(r)-G_{v}^{2}(r)}},
$$

where $G_{a}(r)$ is the average power of the $g(r)$, and $G_{v}(r)$ is the RMS power fluctuation of $g(r)$ over the set $A(r, R)$.

Narrowband Ricean $K$-factor in the viaduct scenario has been analyzed. The CDF of the $K$-factor is shown in Fig. 11. Its median value is $7.63 \mathrm{~dB}$, which is greater than $6 \mathrm{~dB}$ in WINNER II. The fitting of the CDF with a normal distribution and a logistic distribution are also shown in Fig. 11. In order to obtain more accurate fast

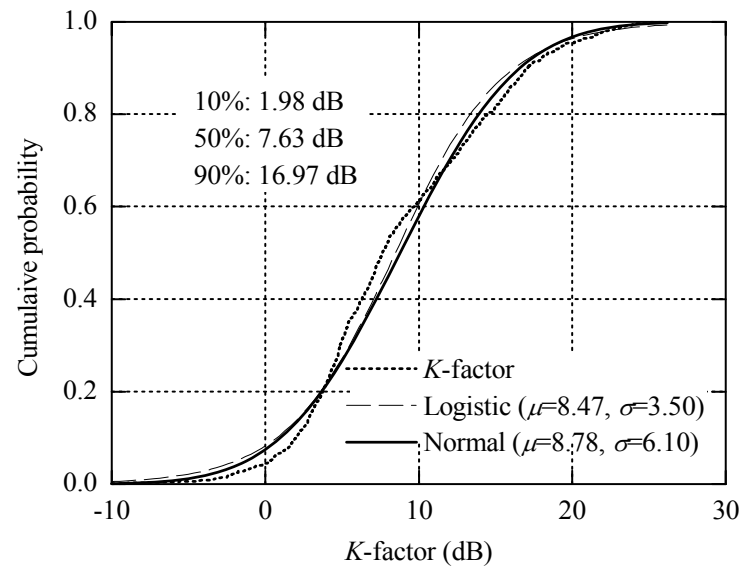

Fig. 11 Distribution of the $K$-factor in a viaduct scenario

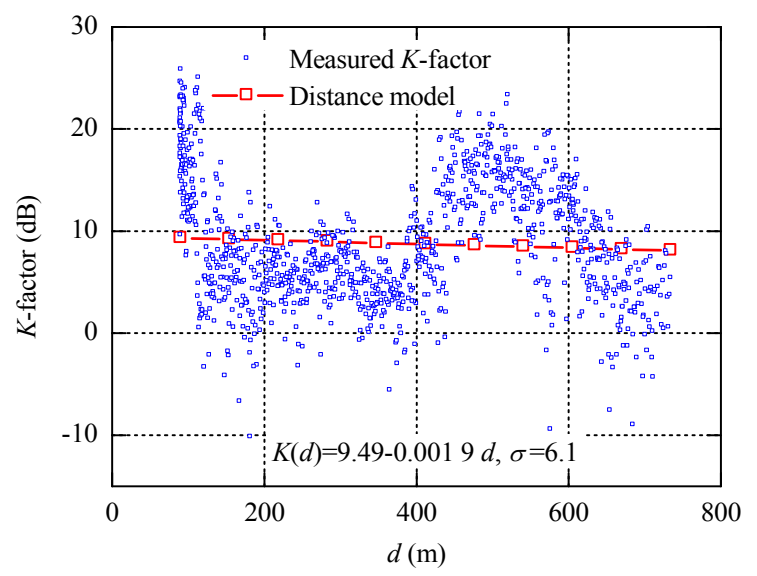

Fig. $12 \mathrm{~K}$-factor as function of distance in a viaduct scenario

variation of the $K$-factor, the estimation interval is set to $1 \mathrm{~m}$. Fig. 12 shows the relationship between $K$-factor and the distance from TX to RX in a viaduct scenario. It can be seen that in this measurement the $K$-factor decreases as the distance increases. The fitting formulas for the $K$ factor is

$$
K=9.49-0.0019 d, \text { with } \sigma=6.1 \mathrm{~dB},
$$

where $d$ is the distance between TX and RX. There is a peak of the Ricean $K$-factor when the distance varies from approximately $400 \mathrm{~m}$ to $600 \mathrm{~m}$. The change of the $K$-factor and the number of resolvable multipath components in Fig. 10 explains each other well: the $K$-factor is bigger when the number of resolvable multipath components is smaller; $K$-factor is smaller when the number of resolvable multipath components is larger.

\subsection{Doppler frequency shift}

In the mobile communication, when the mobile terminal moves to a fixed base station, the receiving frequency becomes higher than the transmitting frequency; when the mobile terminal moves away from the base station, 
the result is the contrary. Doppler frequency shift will make carrier frequency change, and hence has a great influence on the communication system of high-speed railway. A completely analogous characterization of the time-variant nature of the channel can begin in the Doppler shift domain. In this paper we use the method employed in Ref. [10] to obtain the Doppler spectrum. Fig. 13 shows the Doppler frequency shift changing with time. One can see clearly the variation of Doppler frequencies of the strongest paths when the train passes by the TX side. Doppler frequency shift has a dramatic change from a positive value to a negative one. In our measurement, the maximum speed is $196 \mathrm{~km} / \mathrm{h}$ and the Doppler frequency is $426 \mathrm{~Hz}$ at a center frequency of $2.35 \mathrm{GHz}[20]$. The value is very close to that in Fig. 13. The value of power becomes greater when the distance between TX and RX becomes smaller. The Doppler frequency shift changing curve with time forms a $\mathrm{Z}$ shape, which is close to those in Refs. [2] and [10].

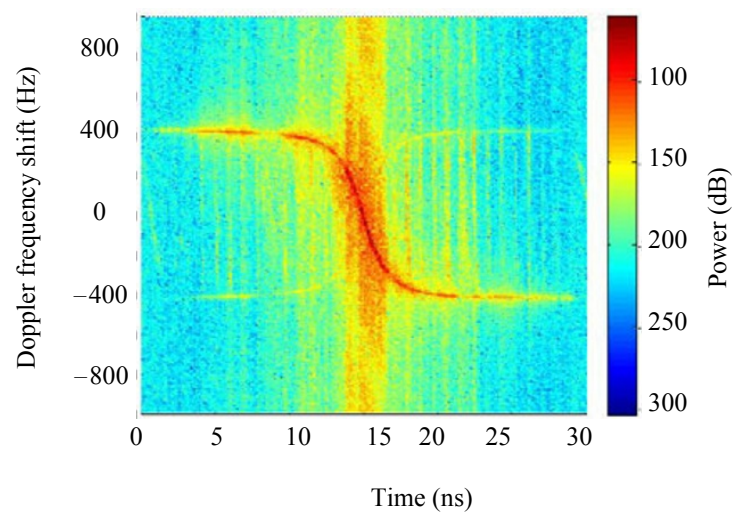

Fig. 13 Doppler transition in a viaduct

\section{Conclusion}

Due to the importance of the channel model in the evaluation and simulation of high-speed railway communication system, a channel measurement is carried out to obtain the channel parameters in a viaduct scenario. A PL model and the PDF of SF are also provided. The estimated PL exponent is 3.56, which is greater than that of the free space model, and the values of PL samples are greater than that of the free space model as well. The reason is that the height of the TX is lower than the height of the RX and the environment around the viaduct in the measurement can intensify the effect. Then the PDF of RMS delay spread and the number of resolvable multipath components are obtained by statistical methods. The mean RMS delay spread obtained from the measurement is greater than that in WINNER II. The CDF of Ricean $K$-factor and the relationship between Ricean $K$-factor and the distance between RX and TX are obtained. The number of resolvable multipath components can explain the changes of Ricean $K$-factor along the measured route very well. The $K$-factor decreases slowly when the distance between TX and RX increases and its median value is $7.63 \mathrm{~dB}$, which is greater than that in WINNER II. Finally, Doppler frequency shift are calculated by the known frequency and velocity, and the calculated maximum Doppler frequency shift is close to that obtained by the measured data.

This paper analyzes the large scale characteristics, RMS delay spread, and Doppler frequency shift of the received wave at the RX side in a viaduct scenario. However, the other small scale characteristics and correlation characteristics are also important to the channel model and need to be investigated in the future. We obtained the correlation of channel parameters from the measured data, but we did not acquire the angle information. Thus, in order to get an accurate channel model, more measurement campaigns should be performed, and our future work is to analyze more parameters of the channel and carry out new measurements.

\section{Acknowledgements}

This research was supported by the National Science \& Technology Pillar Program (No. 2012BAF14B01), the National Natural Science Foundation of China (No. 61171105), and China Mobile Research Institute. The authors thank Weihui Dong from China Mobile Research Institute for his assistance in conducting measurements.

\section{References}

[1] IST-4-027756 WINNER II D1.1.2 v1.2 WINNER II channel models, http://www.ist-winner.org/WINNER II -Deliverables/D1.1.2.zip, 2008-02-04.

[2] R. Parviainen, P. Kyösti, Y Hsieh, et al., Results of high speed train channel measurements, Lille: European Cooperation in the Field of Scientific and Technical Research, 2008.

[3] H. Wei, Z. Zhong, K. Guan, et al., Path loss models in viaduct and plain scenarios of the high-speed railway, In: Proc. of the 5th Int. Communications and Networking in China (CHINACOM) ICST Conf., Beijing, China, Aug. 25-27, 2010: 1-5.

[4] J. Lu, G. Zhu, B. Ai, Radio propagation measurements and modeling in railway viaduct area, In: Proc. of the 6th Int. Wireless Communications Networking and Mobile Computing (WiCOM) Conf., Chengdu China, Sep. 23-25, 2010: 1-5.

[5] H. Wei, Z. Zhong, L. Xiong, et al., Study on the shadow fading characteristic in viaduct scenario of the highspeed railway, In: Proc. of the 6th Int. Communications and Networking in China (CHINACOM) ICST Conf., Harbin, China, Aug. 17-19, 2011: 1216-1220. 
[6] J. Lu, G. Zhu, C. Briso-Rodriguez, Fading characteristics in the railway terrain cuttings, In: Proc. of IEEE 73rd Vehicular Technology Conf. (VTC Spring), Budapest, Hungary, May 15-18, 2011: 1-5.

[7] R. He, Z. Zhong, B. Ai, et al. A novel path loss model for high-speed railway viaduct scenarios, In: Proc. of the 7th Int. Wireless Communications, Networking and Mobile Computing (WiCOM) Conf., Wuhan, China, Sep. 23-25, 2011: 1-4.

[8] R.S. He, Z.D. Zhong, B. Ai, et al., An empirical path loss model and fading analysis for high-speed railway viaduct scenarios, IEEE Antennas and Wireless Propagation Letters, 2011, 10: 808-812.

[9] R. He, Z. Zhong, B. Ai, et al., Propagation measurements and analysis for high-speed railway cutting scenario, Electronics Letters, 2011, 47(21): 1167-1168.

[10] L. Liu, C. Tao, J. Qiu, et al., Position-based modeling for wireless channel on high-speed railway under a viaduct at $2.35 \mathrm{GHz}$, Selected Areas in Communications, 2012, 30(4): 834-845.

[11] Elektrobit Ltd., Propsound multidimensional channel sounder, http://www.propsim.com, 2010-05-01.

[12] D. Dong, J. Zhang, Y. Zhang, et al., Large scale characteristics and capacity evaluation of outdoor relay channels at $2.35 \mathrm{GHz}$, In: Proc. of 2009 IEEE 70th Vehicular Technology Conference Fall (VTC 2009-Fall), Anchorage, AK America, Sep. 20-23, 2009: 1-5.

[13] L. Tian, N. Sheng, F.H. Zhang, et al., Large scale characteristics analysis in microcell to femtocell scenario based on channel measurement, In: Proc. of Wireless Advanced (WiAd) Conference, London, Jun. 20-22, 2011: 122-125.

[14] X. Gao, J. Zhang, G. Liu, et al., Large scale characteristics of $5.25 \mathrm{GHz}$ based on wideband MIMO channel measurements, IEEE Antennas and Wireless Propagation Letters, 2007, 6: 263-266.

[15] J. Zhang, L. Xin, F. Zhang, Propagation characteristics and modeling in indoor multi-floor environment, http://www.sciencedirect.com/science/article/pii/S18744 90712000602, 2012-06-08.

[16] J. Zhang, D. Dong, Y. Liang, et al., Propagation characteristics of wideband MIMO channel in urban micro- and macrocells, In: Proc. of IEEE 19th International Symposium on Personal, Indoor and Mobile Radio Communications (PIMRC), Poznan, Poland, Sep. 15-18, 2008: 1-6.

[17] J. Zhang, D. Dong, Y. Liang, et al., Propagation characteristics of wideband relay channels in urban micro-cell environment, IEEE Antennas and Wireless Propagation Letters, 2010, 9: 657-661.

[18] J. Zhang, Review of wideband MIMO channel measurement and modeling for IMT-advanced systems, Chinese Science Bulletin, 2012, 57(19): 2387-2400.

[19] L.J. Greenstein, D.G. Michelson, V. Erceg, Momentmethod estimation of the Ricean $K$-factor, Communications Letters, 1999, 3(6): 175-176.

[20] Base Station (BS) radio transmission and reception (FDD), 3rd Generation Partnership Project (3GPP), Tech. Rep. 3GPP TS 25.104 V9.4.0, 2010.

(Editor: Dongju CHEN) 